\title{
RAIN IDENTIFICATION IN ASCAT WINDS USING SINGULARITY ANALYSIS
}

\author{
W. Lin ${ }^{(1)}$, M. Portabella ${ }^{(1)}$, A. Stoffelen ${ }^{(2)}$, A. Turiel ${ }^{(1)}$, A. Verhoef ${ }^{(2)}$ \\ ${ }^{(1)}$ Institut de Ciències del Mar-CSIC, Pg. Marítim Barceloneta 37-49, 08003 Barcelona, Spain \\ (2) Royal Netherlands Meteorological Institute (KNMI), Postbus 201, 3730 AE De Bilt, The Netherlands
}

Email:wenminglin@icm.csic.es

\begin{abstract}
The Advanced Scatterometer (ASCAT) onboard the Metop satellite series is designed to measure the global ocean surface wind vector. Generally, ASCAT provides wind products at excellent quality. Occasionally, though, ASCAT-derived winds are degraded by rain. Therefore, identification of rain can help to better understand the rain impact on scatterometer wind quality, and to develop a proper quality control (QC) approach for scatterometer data processing. In this letter, an image processing method, known as singularity analysis (SA), is used to detect the presence of rain such that rain-contaminated wind vector cells (WVCs) are flagged. The performance of SA for rain detection is validated using ASCAT Level-2 data collocated with satellite radiometer rain data. The rain probability as a function of SA singularity exponent is calculated and compared with other rain sensitive parameters, such as the wind inversion residual or maximum-likelihood estimator (MLE). The results indicate that the SA is effective in detecting ASCAT raincontaminated data. Moreover, SA is a complementary rain indicator to the MLE parameter, thus showing great potential for an improved scatterometer QC.
\end{abstract}

\section{INTRODUCTION}

The presence of rain is known to degrade scatterometerderived sea-surface wind quality. Rain drops both attenuate and scatter the microwave signal. Those effects are relevant for Ku-band scatterometers, but relatively small for C-band systems (except for heavy rain conditions). In addition, the splashing of rain alters the wind-induced scatterometer backscatter signature on the ocean surface. At the same time, the wind variability within a wind vector cell (WVC) is enhanced in rain scenarios, which, in turn, increases the measurement variance. If the wind retrieval does not take rain effects into account, the rain contributions are interpreted as wind features, and in turn, the retrieved wind quality is degraded. Over the last decades, several approaches have been proposed to address the mentioned rain effects on scatterometers, especially for Ku-band systems. The first approach consists of identifying the parameters that are sensitive to rain (e.g., retrieved wind speed, MLE, incidence angle and etc.), estimating the rain probability or the retrieved wind quality as a function of those parameters by using a training dataset, and then applying the probability estimation of rain or wind quality indicator to flag data as 'rain-contaminated' [1][2]. The second methodology is based on assessing the rain effects on scatterometer backscatter measurements by using collocated scatterometer wind data, satellite microwavederived rain data, and Numerical Weather Prediction (NWP) wind data, and then correcting the rain-induced backscatter contribution before wind retrieval [3][4]. The third strategy also uses collocated scatterometer/ rain/ NWP wind data to model both wind- and rain-induced backscatter, with the objective of retrieving wind and rain parameters simultaneously [5][6]. Moreover, there are techniques which are based on the use of a single parameter, i.e., the Normalized Radar Cross Section (NRCS, $\sigma^{0}$ ), for rain detection purposes. For instance, the difference between horizontally polarized and vertically polarized $\sigma^{0}$ can be used to define the rain flag [7]. In [8], multi-fractal exponents are computed from the QuikSCAT $\sigma^{0}$ images, and then a threshold is set to separate the rainy cases from the rain-free cases.

For the identical C-band Advanced Scatterometers onboard Metop-A and Metop-B, i.e., ASCAT-A and ASCAT-B, a quality control (QC) based on the wind inversion residual or maximum-likelihood estimator (MLE) is developed to screen the poor-quality winds. A high MLE value corresponds to a poor consistency of the WVC backscatter values, i.e., a low probability that they can be explained by the WVC-mean wind vector. In particular, note that the current operational QC threshold is set for MLE $>+18.6$. The MLE is proved to be sensitive to rain, i.e., it generally increases with rain rate (RR) [9]. However, many WVCs with low MLE values (and therefore not QCed) are also affected by rain (i.e., the retrieved wind quality is degraded). Moreover, the MLE histogram peak of rain-contaminated WVCs is close to that of rain-free cases, which indicates that the MLE itself is ineffective in flagging rain-contaminated ASCAT WVCs in general [9]. Particularly, at low winds backscatter triplets affected by rain may still result in low or negative MLE values.

An image-processing technique, known as singularity analysis (SA), has been recently proposed as a complementary ASCAT QC tool [9]. SA provides quantitative information about the local regularity or irregularity of the signal. It is therefore able to detect not only existing geophysical structures, characterized as singularity fronts, but also any transition due to the presence of retrieval errors. In [9], preliminary results show that SA can potentially be used for ASCAT rain identification.

In this paper, the SA method is further developed for optimizing ASCAT rain identification. In section 2, the concept of singularity analysis is briefly introduced. In section 3, the SA method is applied and optimized for ASCAT data. In section 4 , the experimental results of singularity analysis on rain flagging are presented and a comparison with the MLE-based rain identification is carried out. Finally, the conclusions can be found in section 5 .

\section{SINGULARITY ANALYSIS}

Given a scalar signal $s$, the singularity exponent (SE) $h(\mathbf{x})$ depicts a dimensionless and scale invariant measure of the 
degree of local regularity around a given point $\mathbf{x}$. It can be evaluated according to the following function [10],

$$
\frac{1}{\mathrm{r}}|\mathrm{s}(\mathrm{x}+\mathrm{r})-\mathrm{s}(\mathrm{x})|=\alpha(\mathrm{x}) \mathrm{r}^{\mathrm{h}(\mathrm{x})}+\mathrm{o}\left(\mathrm{r}^{\mathrm{h}(\mathrm{x})}\right)
$$

where $\alpha(\mathrm{x})$ is a dimensional and signal-dependent amplitude factor, and the factor $\mathrm{o}\left(r^{h(\mathbf{x})}\right)$ becomes negligible when $r$ goes to zero. The left part of Eq. (1) is the gradient estimated at half the radius $r$. Therefore, the singularity exponent roughly behaves as,

$$
h(\mathbf{x}) \sim \frac{\log |\nabla s(\mathbf{x})|}{\log r}
$$

Since the presence of long-range correlations in real data can mask the value of $h(\mathbf{x})$, a wavelet projection is used to filter the signal and to provide a stable interpolation scheme in a continuous range of scales. Given a wavelet $\Psi(\mathbf{x})$, the wavelet projection of Eq. (1) becomes

$$
\mathrm{T}_{\Psi}|\nabla s|(\mathbf{x}, r)=\alpha(\mathbf{x}) r^{h(\mathbf{x})}+\mathrm{o}\left(r^{h(\mathbf{x})}\right)
$$

So the singularity exponent corresponds to,

$$
h(\mathbf{x}) \sim \frac{\log \mathrm{T} \Psi|\nabla s|(\mathbf{x}, r)}{\log r}
$$

Since we are mainly interested in the most singular structures, the singularity exponents can be estimated in the following way to avoid projecting across multiple scales,

$$
h(\mathbf{x})=\frac{\log \left(\mathrm{T} \Psi|\nabla s|\left(\mathbf{x}, r_{0}\right) /\left\langle\mathrm{T} \Psi|\nabla s|\left(\mathbf{x}, r_{0}\right)\right\rangle\right)}{\log r_{0}}+\mathrm{o}\left(\frac{1}{r_{0}}\right)
$$

where $\left\langle\mathrm{T}_{\Psi}|\nabla s|\left(\mathbf{x}, r_{0}\right)\right\rangle$ is the mean value of the wavelet projection over the whole signal. The scale $r_{0}$ is defined as the smallest accessible scale, i.e., one pixel scale for a discrete 2D image. The numerical implementation of Eq.(5) is described in [11]. Negative singularity exponents derived from Eq. (5) depict that the function is less regular, while the positive values indicate a more regular behavior. SA can be applied to any satellite-derived image. In this paper, SA is applied to ASCAT data.

\section{SA APPLICATION TO ASCAT DATA}

The SA algorithm needs be adapted before its application on ASCAT data. All the ASCAT-derived parameters, such as the $\sigma^{0}$ measurements, the inversion residual (MLE), the measurement variability parameter (i.e., $K_{\mathrm{p}}$ as estimated in [12]), and the retrieved wind components (i.e., U, V, speed and direction) can be used to generate singularity maps. Different singularity maps are used for different purposes. Thus, SA optimization is required for rain identification.

\subsection{Adaption of SA to ASCAT data}

In general, the SA algorithm described in [11] works well on ASCAT data. However, it shows in [13] that SA overestimates the irregularity (very negative singularity exponents) of ASCAT measurements at the edge of the swath. A similar effect is found at the edges of non-ocean areas, such as, coastlines, islands, sea ice margin. To overcome such drawbacks, the following processing is performed prior to SA:

a) Within the ASCAT image, the meaningless non-ocean WVC values are replaced by the mean of the input ASCAT parameters over all ocean WVCs within a centered $3 \times 3$ box. If the non-ocean WVC is at the corner (or edge) of the image, a closest $2 \times 2$ (or $3 \times 2$ ) box is used; $b$ ) The image is extended to the left and right sides of the swath by one node at each row, and the extrapolation point is filled using the mean of ASCAT parameter over all ocean WVCs within the closest $3 \times 2$ box (or $2 \times 2$ box in case the extrapolation is performed at a swath corner point);

c) The image is also extended before the first row of WVCs and after the last row of WVCs by one point at each column, and the extrapolation point is filled using the mean of ASCAT parameter over all ocean WVCs within the closest $2 \times 3$ ( $2 \times 2$ for corner WVCs) box.

Considering the study on ASCAT $12.5-\mathrm{km}$ product, each image consists of $\sim 100 \times 41 \mathrm{WVCs}$, corresponding to a 3 min long set of WVC rows from one individual swath. Such pre-processing steps remove potential edge artefacts in SA while preserving the information content of the image elsewhere (e.g., singularity fronts associated with rain). As such, SA is applied on the preprocessed image using Eq.(3). And, SE is computed over all ocean WVCs of the original input image.

Due to the noisy nature of the ASCAT parameters (for example over rainy areas), there may be isolated extremely positive or negative SE values after singularity analysis. Therefore, the mean SE value within a centered $3 \times 3$ window, i.e., $3 \times 3 \mathrm{WVCs}$, is taken to generate the final singularity exponent for each WVC. If the ocean WVC is at the corner (or edge) of the image, a closest $2 \times 2$ (or $3 \times 2$ ) box is used.

\subsection{SA optimization for rain identification}

As already mentioned, all the ASCAT-derived parameters can be used to generate singularity maps. In [13], the lowest (most negative) singularity exponents from the singularity maps of the ASCAT zonal $(u)$ and meridional (v) wind components are used to generate the singularity map. Singularities indeed appear in areas of wind discontinuities, i.e., convergence or divergence corresponding to fronts or downbursts (as observed by ASCAT), that may be associated with rain. In this section, other rain-sensitive ASCAT parameters are assessed before choosing the most optimal singularity maps for rain identification over the ocean surface. As an example, Figure 1(a) shows a particular ASCAT-retrieved wind field (ASCAT 12.5-km product, observed at 20:30 September 24, 2008) with the Tropical Rainfall Measuring Mission's (TRMM) Microwave Imager (TMI) collocated rain rate values superimposed. The collocation criteria for TMI rain data are less than 30 min distance in time and 25 $\mathrm{km}$ distance in space from the ASCAT measurements. Typical wind responses, including increased wind variability and wind fronts, are found in the rainy areas. Figures $1(b)$ and $(c)$ illustrate the corresponding MLE and $K_{\mathrm{p}}$ (i.e., the mean $K_{\mathrm{p}}$ of the fore and the aft beams) values. At low wind speed conditions, the raindrop "splashing" causes additional roughening of the sea surface, and in turn, an increase of the ocean backscatter from scatterometers. This results in a remarkable increase of the retrieved wind speed. In contrast, at high wind speed conditions, the rain splashing effect is relatively small. Therefore, singularity exponents of the wind speed field can be used to assess the irregularities associated with low wind speed conditions (Fig. 1, areas around $\left[164.5^{\circ} \mathrm{W}\right.$ $\left.18.5^{\circ} \mathrm{S}\right]$ and $\left.\left[161.5^{\circ} \mathrm{W} 21.5^{\circ} \mathrm{S}\right]\right)$. In order to detect the wind discontinuities associated to the rain-induced flow, SA is then applied on the wind direction field. Singularities can be detected over the sharp transition areas (Fig. 1(a), the 


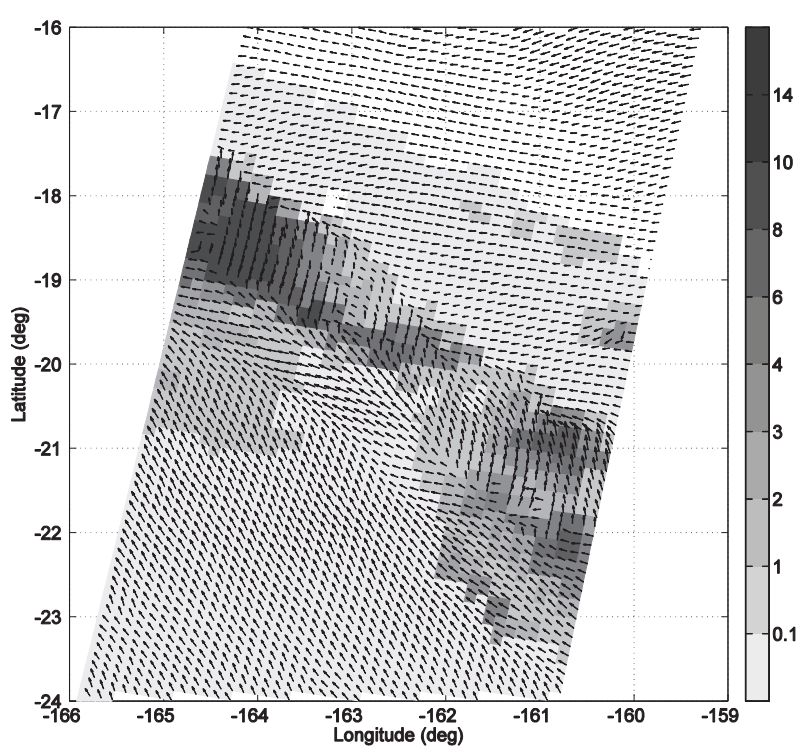

(a)

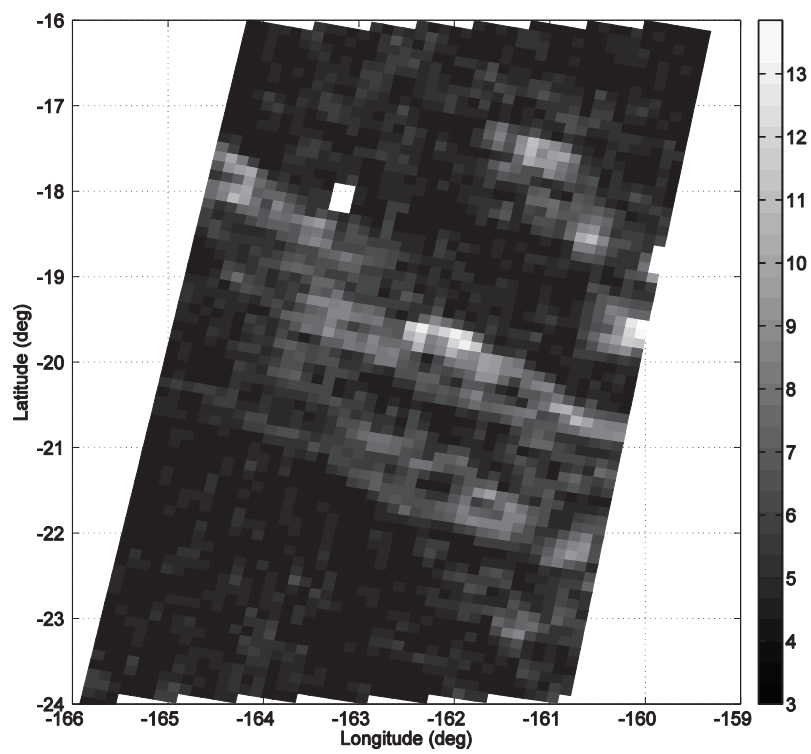

(c)

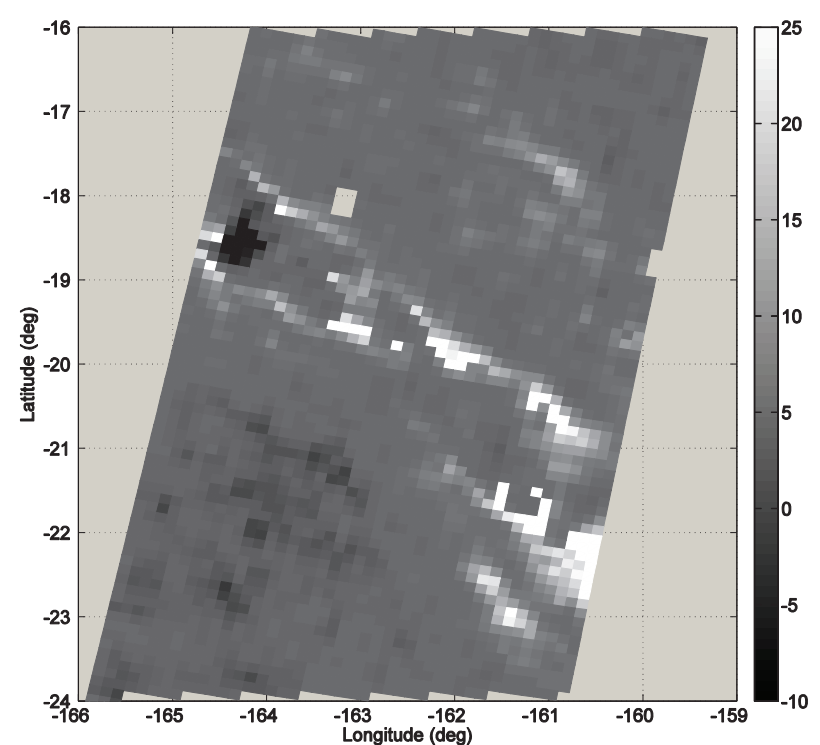

(b)

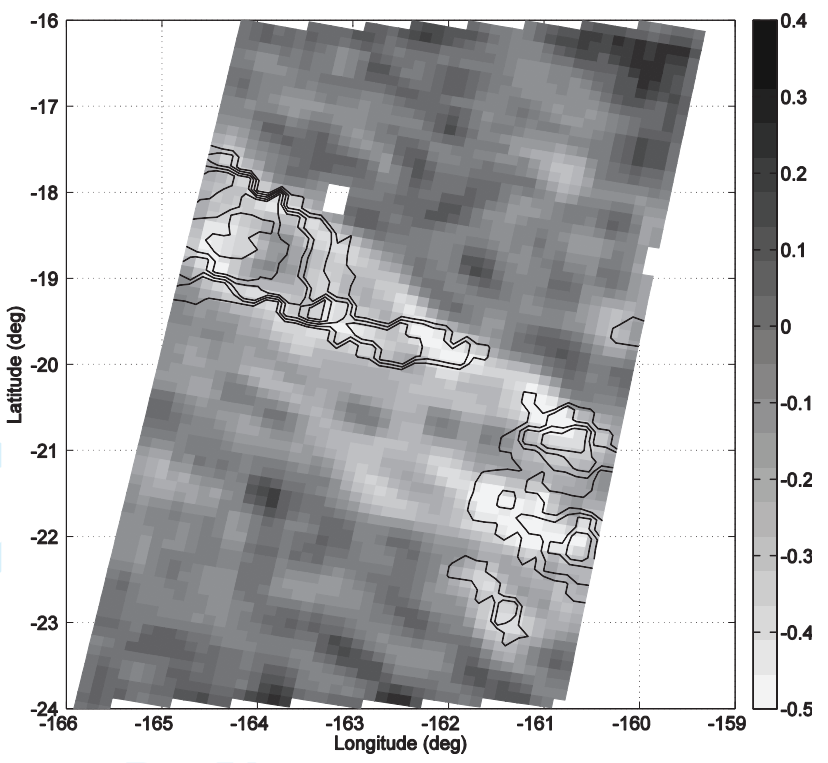

(d)

Fig. 1(a) Map of collocated ASCAT-TMI data. The grayscale square areas superimposed correspond to different TMI RRs (see the legend, mm/hr). Note that the white background corresponds to no TMI RR data. The acquisition date was September 24, 2008, at 20:30 UTC; (b) the corresponding MLE distribution and $(c) K_{\mathrm{p}}$ (mean value of fore and aft beams, \%) distribution; $(d)$ Singularity map of the ASCAT-retrieved wind field shown in Fig. 1 $(a)$. At every grid point, the minimum SE value from the wind speed, wind direction, and MLE SEs is used to generate the map. The RR contour lines depict the rainy areas.

convergence goes from $\left[164^{\circ} \mathrm{W} 18^{\circ} \mathrm{S}\right]$ to $\left.\left[160.5^{\circ} \mathrm{W} 21^{\circ} \mathrm{S}\right]\right)$. Meanwhile, the rain-impacted ASCAT measurements are generally more inconsistent with the empirical Geophysical Model Function (GMF) than rain-free measurements. This inconsistency results in an increase of the inversion residual and a decrease of the retrieved wind quality. An MLE sign has been defined in [14] to improve the ASCAT MLE-based QC. For low wind speed conditions and in case of heavy rain, the measured $\sigma^{0}$ triplets are generally located outside the cone surface [15] (i.e., negative MLE values) as defined by the GMF. Therefore, the singularity exponent of the MLE field is also examined to better identify rain.

Regarding the measurement variability factor, as the wind variability within a certain $\mathrm{WVC}$ increases with rain rate, $K_{\mathrm{p}}$ value increases with RR in general (see the white areas in Fig. 1(c)). However, at low wind speed conditions, high $K_{\mathrm{p}}$ value are also found due to the high wind variability (see the area around $\left[161^{\circ} \mathrm{W} 18^{\circ} \mathrm{S}\right]$ in Fig. $1(c)$ ). Moreover, the estimation of $K_{\mathrm{p}}$ is rather noisy as indicated by the granularity of the $K_{\mathrm{p}}$ map, thus making the rain signature in $K_{\mathrm{p}}$ less evident. As such, the singularity map of $K_{\mathrm{p}}$ is not used in this study.

In this paper, singularity maps of the inversion residual and the retrieved wind components (speed and direction) are examined independently for the particular wind field. Then at every grid point, the minimum SE value from the wind speed, wind direction, and MLE SEs is used to generate the final singularity map. Through this approach, the wind discontinuities (convergence or divergence) associated with the rain-induced flow and the patchy structure of rainy areas (artefacts due to rain contamination) can be detected by singularity analysis simultaneously. Figure $1(d)$ shows the singularity map corresponding to the ASCAT wind and MLE fields shown in Figs. 1(a) and 1(b). It is also clear that the TMI rain contours in Fig. 1(d) well corresponds with the negative SE values. Note that the comparison between SE and TMI RR is intended only to validate the presence of rain, but not the rain rate. 

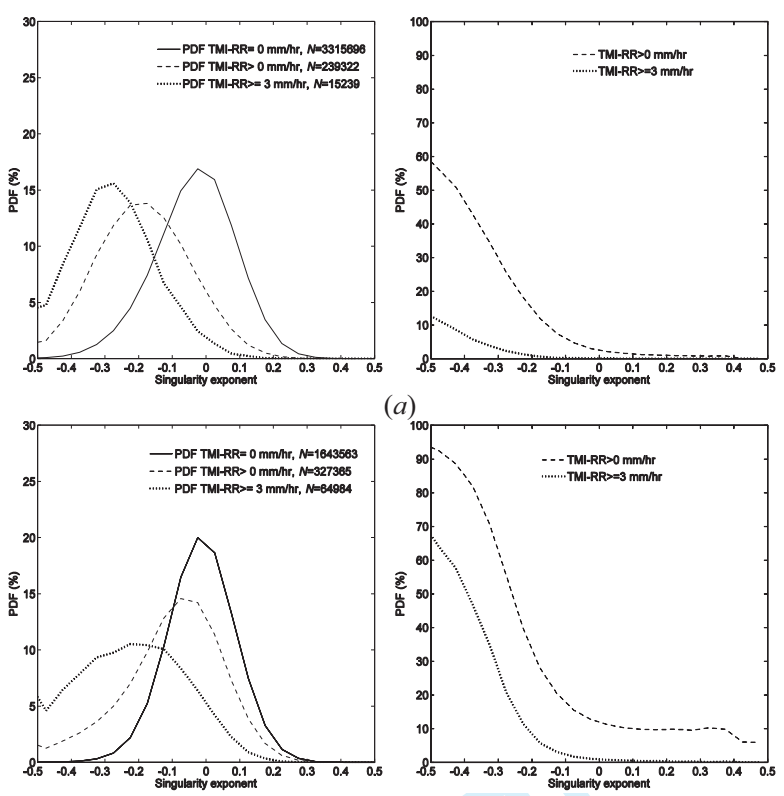

(a)

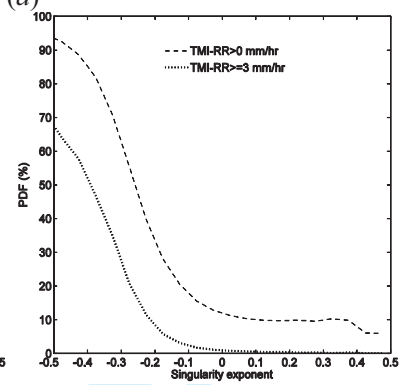

(b)

Fig. 2 Illustrations of the PDF of $p(\mathrm{SE} \mid R)$ (left panels) and the rain probability $p(R \mid \mathrm{SE})$ (right panels) for $(a)$ : low wind speeds $(4 \leqslant V<6 \mathrm{~m} / \mathrm{s})$ and $(b)$ : high wind speeds $(V \geqslant 10 \mathrm{~m} / \mathrm{s}$ ) conditions respectively

\section{EXPERIMENTAL RESULTS}

To estimate the probability of rain $(\mathrm{R}) p(R)$ as a function of SE, 8 months (from September 2008- May 2009) of collocated ASCAT 12.5-km product, TMI RR and European Centre for Medium-range Weather Forecasts (ECMWF) winds are explored in this study. ECMWF winds are acquired by interpolating three surrounding ECMWF forecast winds (selected from $+3 \mathrm{~h}$ to $+18 \mathrm{~h}$ forecast range in 3-hour steps) on a $62.5-\mathrm{km}$ grid both spatially and temporally to the ASCAT data acquisition location and time, respectively. $p(R)$ is estimated by accumulating two histograms. The first histogram contains the total number of WVCs in the studied category. The second one contains the number of rain-affected WVCs. By dividing the second histogram by the first one, we obtain an estimate of the rain probability.

Figure 2(a) illustrates the PDF of $p(\mathrm{SE} \mid R)$ (left panel) and the rain probability $p(R \mid \mathrm{SE})$ (right panel) for low wind speeds $(4 \leqslant V<6 \mathrm{~m} / \mathrm{s})$. Figure $2(b)$ shows the same plots for high wind speeds $(V \geqslant 10 \mathrm{~m} / \mathrm{s})$. Two kinds of rain conditions, i.e., TMI-RR $>0 \mathrm{~mm} / \mathrm{hr}$ and TMI-RR $\geqslant 3$ $\mathrm{mm} / \mathrm{hr}$, are studied. There is an increasing shift of the SE distributions towards negative SE values with increasing RR. The PDF peak of rain-contaminated WVCs is distinct from that of rain-free cases. As noted by the difference between the dashed and the dotted curves in the right panel of Fig. 2(a), the anomalies associated with negative SE values are generally associated with light rain contamination at low wind speeds condition. However, at high winds, such anomalies are associated with heavy rain contamination (see right panel of Fig. 2(b)).

For comparison, Fig. 3 shows the same PDFs as Fig. 2 but for the MLE parameter. At low and high wind speed conditions, the extreme (positive or negative) MLE values are generally associated with heavy rain contamination (TMI-RR $\geqslant 3 \mathrm{~mm} / \mathrm{hr}$ ). At low winds, heavy rain contamination is mainly present at large negative MLE values (measurement triplets located outside the cone surface [14]), whereas at high winds, it is present at large
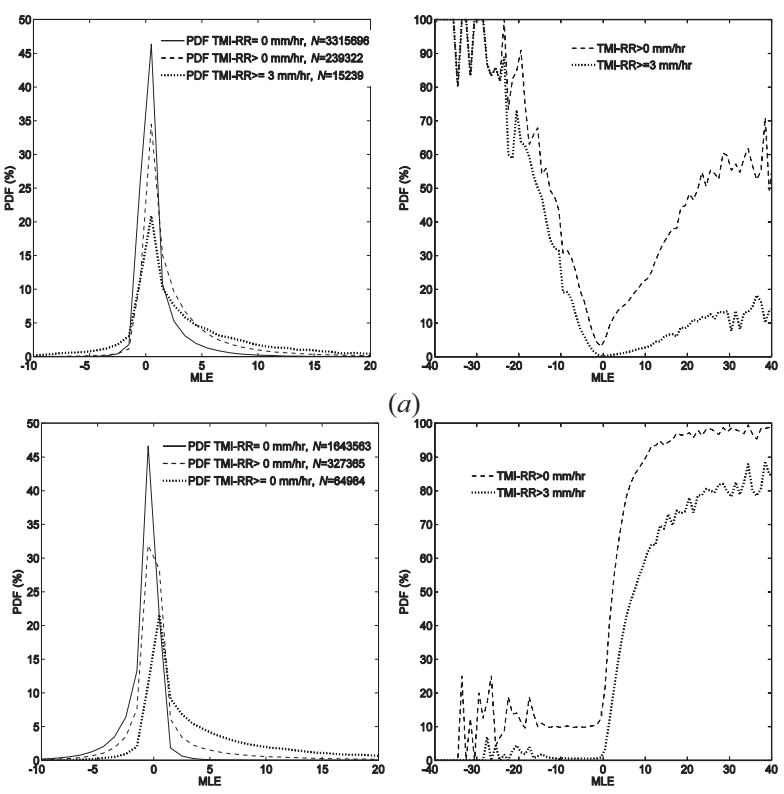

(b)

Fig.3 Illustrations of the PDF of $p(\operatorname{MLE} \mid R)$ (left panels) and the rain probability $p(R \mid \mathrm{MLE})$ (right panels) for $(a)$ : low wind speeds $(4 \leqslant V<6$ $\mathrm{m} / \mathrm{s})$ and $(b)$ : high wind speeds $(V \geqslant 10 \mathrm{~m} / \mathrm{s})$ conditions respectively

Table 1. Statistics of the ASCAT rain flagging using the singularity analysis (SE threshold: -0.45) and the operational MLE-based (MLE $>+18.6)$ methods. The second and third rows show the vector rootmean-square (VRMS) differences between the ASCAT winds and the ECMWF winds. The last two rows present the percentage of flagged WVCs contaminated by rain, according to different TMI RR intervals.

\begin{tabular}{|l|c|c|}
\hline & SE & MLE \\
\hline Flagging ratio (\%) & 0.42 & 0.31 \\
\hline VRMS, Non-flagged (m/s) & 2.28 & 2.28 \\
\hline VRMS, flagged (m/s) & 5.91 & 6.07 \\
\hline$\%$ of flagged WVCs with TMI-RR $>0 \mathrm{~mm} / \mathrm{hr}$ & 82.3 & 72.7 \\
\hline$\%$ of flagged WVCs with TMI-RR $\geqslant 3 \mathrm{~mm} / \mathrm{hr}$ & 44.5 & 46.0 \\
\hline
\end{tabular}

positive MLE values (triplets located inside the cone).

From the right panels, it seems appropriate to set a MLE threshold to separate rainy cases from rain-free ones. However, since the PDF peaks of rain-free WVCs and rain-contaminated WVCs are quite close to each other (as shown in the left panels, the peaks are around MLE $=0$ ), a low MLE threshold will cause substantial false alarm rate. In contrast, since SE distributions shift considerably with RR (see left panels of Fig. 2), setting a SE threshold can be very effective in filtering rain while keeping a low false alarm rate for low winds.

Table 1 shows the statistics of SE-based and MLE-based flags respectively. An SE threshold of -0.45 is used, i.e., WVCs with SE<-0.45 are flagged. The threshold of the MLE-based flag is that used in the operational QC, i.e., WVCs with MLE $>+18.6$ are flagged. For the given thresholds, singularity analysis flags a bit more raincontaminated WVCs than MLE. However, the latter flags slightly more heavy rain contaminated cases than SA. Another interesting result is that WVCs flagged by MLEbased filter and WVCs flagged by SE-based filter do generally not coincide (coincidence ratio less that $20 \%$ for the given thresholds in table 1), indicating that SE is not only a good rain indicator but also very complementary to the operational MLE-based QC. The VRMS differences between the ASCAT winds and the ECMWF winds are also presented in the table to validate the different flags in terms of wind quality. It shows that SA also detects poorquality WVCs. 
Note that both flags filter a small portion of poor-quality WVCs in the absence of rain. These poor-quality winds are caused by increased local wind variability, confused sea state, and/or radar footprint contaminated by land or ice, which increase the measurement variance, and lead to large discrepancies between the measured triplets and the GMF (i.e., high MLE values), and low negative SE values. In the presence of rain, the associated rain splash and wind downbursts change the characteristics of the SE and MLEs and which is the focus of this paper.

\section{CONCLUSIONS}

In this study, a new image processing technique, the socalled singularity analysis, is adapted for ASCAT rain detection. It is shown that SA successfully exploits the rain information content (rain signatures) present in the different ASCAT parameters, and thus an effective raincontamination detection tool emerges. Moreover, SA mostly detects rain-contaminated WVCs when the MLEbased QC does not, and vice versa, indicating that both techniques are very complementary in terms of both rain detection and quality control.

SA uses the information present in the ASCAT data itself and, as such, is useful for both ASCAT near-real-time products and offline products. The SA code has been optimized for near-real-time processing.

Further developments are needed in order to better separate actual wind discontinuities (e.g., fronts and downbursts) from rain-induced discontinuities (i.e., artefacts due to rain contamination). Moreover, in order to improve the ASCAT rain flag and quality control, a combined analysis of SE, MLE and other ASCAT rain/quality-sensitive parameters will be investigated.

\section{ACKNOWLEDGEMENTS}

This work was supported in part by the European Organisation for the Exploitation of Meteorological Satellites (EUMETSAT) Ocean and Sea Ice Satellite Application Facility (SAF) Associated Scientist project under Reference OSI-AVS-12-04 and in part by the Spanish Ministry of Science and Innovation (MICINN) National R\&D project under Reference AYA2012-39356C05-03. The ASCAT level $1 \mathrm{~b}$ data are provided by EUMETSAT. The software used in this paper was developed through the EUMETSAT Numerical Weather Prediction SAF. The ECMWF data were retrieved from the ECMWF MARS archive. The TMI data are available from the Web site of Remote Sensing Systems (http://www.ssmi.com/). The authors greatly appreciate the three reviewers who helped to improve the paper.

\section{REFERENCES}

[1] J. N. Huddleston and B. W. Stiles, "A multidimensional histogram technique for flagging rain contamination on QuikSCAT", in Proc.IEEE Int. Geosci. Remote Sens. Symp., Honolulu, HI, 2000, vol. 3,pp. 12321234.

[2] M. Portabella and A. Stoffelen, "A comparison of KNMI quality control and JPL rain flag for SeaWinds", Can. J. Remote Sens., vol. 28, no. 3, pp. 424-430, 2002.

[3] B. W. Stiles and S. H. Yueh, "Impact of rain on spaceborne Ku-band windscatterometer data", IEEE Trans.
Geosci. Remote Sens., vol. 40, no. 9,pp. 1973-1983, Sep. 2002.

[4] K. A. Hilburn, F. J. Wentz, D. K. Smith, and P. D. Ashcroft, "Correcting active scatterometer data for the effects of rain using passive microwave data", J. Appl. Meteorol. Climatol., vol. 45, no. 3, pp. 382-398,Mar. 2006. [5] D. W. Draper and D. G. Long, "Simultaneous wind and rain retrievalusing SeaWinds data", IEEE Trans. Geosci. Remote Sens., vol. 42, no. 7,pp. 1411-1423, Jul. 2004.

[6] C. Nie and D. G. Long, "A C-band scatterometer simultaneous wind/rain retrieval method", IEEE Trans. Geosci. Remote Sens., vol. 46, no. 11,pp. 3618-3632, Nov. 2008.

[7] D. E. Weissman, M. A. Bourassa, J. J. O’ Brien, J. S. Tongue, "Calibrating the QuikSCAT/SeaWindsRadar for Measuring Rain rate Over the Oceans", IEEE Trans Geosc and Rem. Sens., vol. 41, No.12, pp.2814-2820, Dec. 2003

[8] V. G. Torsekar, "Oceanic rain identification using multi-fractal analysis of QuikSCAT sigma-0", Master Thesis, University of Central Florida, Orlando, 2005.

[9] M. Portabella, A. Stoffelen, W. Lin, A. Turiel, A. Verhoef, J. Verspeek, and J. Ballabrera, "Rain effects on ASCAT wind retrieval: Towards an improved quality control", IEEE Trans. Geosci. Remote. Sens., vol. 50, no. 7, pp.2495-2506, 2012.

[10] O. Pont, A. Turiel, and C.J. Perez-Vicente, "Empirical evidences of a common multifractal signature in economic, biological and physical systems", Physica A, 388:2025-2035, 2009.

[11] O. Pont, A. Turiel, and H. Yahia, "An optimized algorithm for the evaluation of local singularity exponents in digital signals", in: Aggarwal, J., Barneva, R., Brimkov,V., Koroutchev, K., Korutcheva, E. (eds.) 14th International Workshop, IWCIA2011. Lecture Notes in Computer Science (LNCS), vol. 6636, pp. 346-357. Springer, Madrid, Spain (2011). Available: http://hal.inria.fr/inria-00581057/en/

[12] C. Anderson, H. Bonekamp, C. Duff, J. Figa-Saldaña, and J.J.W. Wilson, "Analysis of ASCAT ocean backscatter measurement noise", IEEE Trans. Geosci. Remote. Sens., vol. 50, no. 7, pp.2449-2457, 2012.

[13] A. Turiel, M. Portabella, W. Lin, and J. BallabreraPoy, "Quality assessment of ASCAT wind vector maps through singularity analysis", SMOS BEC Technical Note, BEC-TN.2012.02 v.1, Feb. 2012. [Online] Available: www.smos-bec.icm.csic.es/publications

[14] M. Portabella, A. Stoffelen, A. Verhoef, and J. Verspeek, "A new method for improving scatterometer wind quality control", IEEE Trans. Geosci. Remote. Sens. Lett., 9, 579-583, 2012.

[15] A. Stoffelen, and D. Anderson, "Scatterometer data interpretation: measurement space and inversion,"J. Atmos. Ocean. Technol., 14(6), 1298-1313, 1997. 